group in contrast to the MTX group, where micronucleus count was elevated as compared to the vehicle control group. Conclusions The CIA study in rats indicates that the MTX prodrug ROS101 may be efficacious for the treatment of RA at an equimolar dose compared to MTX, while avoiding adverse effects known to restrict treatment with MTX.

Acknowledgements C.A. Hansen, co-founder of ROS Therapeutics.

Disclosure of Interest V. Previtali: None declared, N. Woodworth: None declared, M. Hopkins: None declared, J. Petersen Shareholder of: ROS Therapeutics, Consultant for: ROS Therapeutics, I. Ahnfelt-Rønne Shareholder of: ROS Therapeutics, Consultant for: ROS Therapeutics, M. Clausen Shareholder of: ROS Therapeutics.

\section{P144 EFFECT OF LOW-DOSE IONIZING RADIATION ON THE INFLAMMATORY PHENOTYPE OF ADIPOCYTES AND DIFFERENTIATION OF OSTEOCLASTS (IN VITRO)}

K Shreder*, F Rapp, D Kraft, S Hassan, C Fournier. GSI Helmholtzzentrum Für Schwerionenforschung Gmbh, Darmstadt, Germany

10.1136/annrheumdis-2018-EWRR2019.127

Career situation of first and presenting author Post-doctoral fellow.

Introduction Adipose tissue is a complex endocrine organ that produces a variety of immune and inflammatory mediators. Adipocytes, the dominant cell type of adipose tissue, are known to support inflammatory processes in muskuloskeletal diseases such as rheumatoid arthritis (RA) and osteoarthritis (OA) by release of different cytokines and adipokines. During this process, osteoclastogenesis is also enhanced and results in an imbalance of bone metabolism. Low-dose radiation therapy (LD-RT) is known to attenuate inflammation and to increase the mobility of patients suffering from RA or OA. ${ }^{1}$

Objectives In our previous work, we observed a decrease of visfatin levels in serum of patients treated with low-dose ionizing radiation during exposure to the alpha-emitter radon. ${ }^{2}$ In the same study, a decrease of markers for bone resorption after radon exposure was detected. Based on this, we next compared the response of human adipocytes derived from subcutaneous and infrapatellar adipose tissue to ionizing radiation with respect to release of adipokines and other inflammatory factors (IL-6, IL-8). In parallel, we analyzed the effect of ionizing radiation on differentiation capacity of osteoclast (OC) precursors into mature, bone resorbing OC.

Methods Human subcutaneous predipocytes and human infrapatellar preadipocytes were irradiated with different doses of ionizing radiation, and release of inflammatory factors was measured in the cell culture supernatants using ELISA. OC precursors were isolated from human donor blood, differentiated according to standard protocols and analyzed by fluorescent stainings for cell nuclei, tartrate-resistant acidic phosphatase (TRAP) and actin filaments.

Results The results revealed that the release of adipokines and inflammatory cytokines (IL-6, IL-8) was not significantly affected by ionizing radiation. Further, it was found that differentiation of OC precursor cells into mature OC is reduced after irradiation.

Conclusions The observations made in this study suggest that adipocytes are probably not the main source of modified adipokine levels in the arthritic joint. However, an observed tendency of adipocytes to increase fat accumulation after irradiation suggests radiation-induced changes in functionality of human adipocytes which could have an indirect impact on the radiation response of the tissue. OC respond to radiation by reduced differentiation and structural changes, but the impact on functionality needs to be further tested.

\section{REFERENCES}

1. Frey $\mathrm{B}$, et al. Cancer Lett 2015;368:230-237.

2. Cucu A, Shreder K, et al. Front Immunol 2017:8:1-9.

Acknowledgements German Federal Ministry of Education and Research (02NUK017A).

Disclosure of Interest None declared.

\section{P145 HCQ ALLEVIATES 5-FU-INDUCED INTESTINAL INFLAMMATION THROUGH INHIBITING TLR9- DEPENDENT DNA SENSING PATHWAY}

${ }^{1} \mathrm{X} \mathrm{Li},{ }^{2} \mathrm{Y}$ Sun, ${ }^{1} \mathrm{~J}$ Zhang, ${ }^{1} \mathrm{~J} \mathrm{He},{ }^{1} \mathrm{C} \mathrm{Yu},{ }^{1} \mathrm{H}$ Jie, ${ }^{1} \mathrm{E}$ Sun*. 'Department of Rheumatology and Immunology, The Third Affiliated Hospital, Southern Medical University, Guangzhou; ${ }^{2}$ Department of Rheumatology and Immunology, The Second Affiliated Hospital of Soochow University, Suzhou, China

\subsection{6/annrheumdis-2018-EWRR2019.128}

Career situation of first and presenting author Young investigator.

Introduction Evidences revealed that chemotherapies could trigger DNA release, then induce inflammation of intestinal tissues which damp the effect of anti-cancer treatment. ${ }^{1}{ }^{2}$ DNA released induces the translocation of TLR9 to endolysosomes and subsequent nuclear factor- $\kappa \mathrm{B}(\mathrm{NF}-\kappa \mathrm{B})$ activation,

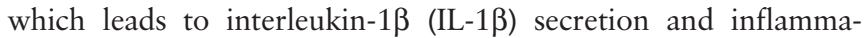
tion. ${ }^{3}$ Targeting TLR9-dependent DNA sensing pathway may be a valuable therapy for chemotherapy induced intestinal mucositis.

Objectives This study aims to investigate whether hydroxychloroquine (HCQ), suppresses 5-FU-induced intestinal mucositis through inhibiting TLR9-dependent DNA sensing pathway. Methods The effect of HCQ on 5-FU-induced intestinal mucositis were examined in vivo and in vitro. We established 5-FUinduced intestinal mucositis model and assessed body weight, diarrhea score and histopathologic changes following HCQ treatment in vivo, then TLR9 and NF- $\mathrm{KB}$ expression of small intestine and IL-1 $\beta$ secretion of serum were analyzed. Bone marrow-derived macrophages (BMDMs) were cultured, transfected with calf-thymus DNA(CT-DNA) and treated with HCQ for 6 hour in vitro. TLR9 and NF- $\kappa \mathrm{B}$ expression and IL-1 $\beta$ secretion in BMDMs were then investigated.

Results HCQ treatment markedly attenuated body weight loss, severity of diarrhea, intestine shortening, and destruction of small intestinal in histopathology of 5-FU- treated mice in vivo. Also HCQ treatment inhibited TLR9 and NF- $\mathrm{KB}$ expression in small intestine of 5-FU- treated mice and pro-inflammatory IL-1 $\beta$ secretion in serum of 5 -FU- treated mice. Meanwhile administration of HCQ reduced the number of macrophages in small intestine of 5-FU-treated mice. Then BMDMs were cultured, transfected with CT-DNA and treated with HCQ in vitro. We found HCQ efficiently inhibited TLR9 expression, translocation of NF- $\mathrm{KB}$ to nucleus, and IL$1 \beta$ secretion in supernatant of CT-DNA stimulated BMDMs.

Conclusions These results provides a new insight into the mechanism of chemotherapy induced intestinal mucositis and 
indicate HCQ may be used as a strategy against intestinal mucositis during 5-FU chemotherapy.

\section{REFERENCES}

1. Woo S-R, Fuertes MB, Corrales L, et al. STING-Dependent Cytosolic DNA Sensing Mediates Innate Immune Recognition of Immunogenic Tumors. Immunity 2014;41:830-42.

2. Hu B, Jin C, Li H-B, et al. The DNA Sensing Aim2 Inflammasome Controls Radiation Induced Cell death and Tissue Injury. Science (New York, NY). 2016;354:765-8.

3. Miura K, Kodama Y, Inokuchi S, et al. Toll-Like Receptor 9 Promotes Steatohepatitis by Induction of Interleukin-1 $\beta$ in Mice. Gastroenterology. 2010;139:323-34. e7.

Acknowledgements The authors confirm that there are no conflicts of interest.

Disclosure of Interest None declared.

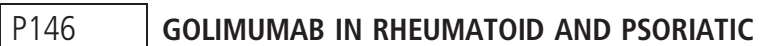 ARTHRITIS. THE RELATIONSHIP BETWEEN MINIMUM SERUM CONCENTRATION, ANTI-GOLIMUMAB ANTIBODIES AND ACTIVITY OF DISEASE}

IJ Uceda*, ${ }^{2} \mathrm{MJ}$ Fobelo, ${ }^{3} \mathrm{R}$ Hernandez. ${ }^{1}$ Rheumatology Unit; ${ }^{2}$ Pharmacy Unit, University Valme Hospital; ${ }^{3}$ hheumatology Unit, University Valme Hospital, Seville, Spain

10.1136/annrheumdis-2018-EWRR2019.129

Career situation of first and presenting author Young investigator.

Introduction Currently there is very little information on optimal levels of golimumab in patients with polyarthritis.

Objectives The main objective was to analyze the minimal serum concentration of golimumab (GOL), anti-golimumab antibodies (AcGOL) and its relation to the activity of the disease measured by DAS 28 index, in rheumatoid arthritis (RA) and psoriatic arthritis (PA).

Methods We conducted a retrospective observational study with RA or PA patients on golimumab treatment between 2011 and 2016.

We analized the age, sex, diagnosis, dose, dose interval, duration of treatment, concomitant disease modifying drugs (DMARs), minimum concentration of GOL (GOL Cmin), AcGOL and DAS28 levels. Sampling was performed on the day corresponding to the dose of golimumab, prior to administration, in order to obtain the minimum levels of the drug. The analytical technique used for the determination was the Promonitor ${ }^{\circledR}$ sandwich ELISA.

Results 10 RA, 5 AP patients were selected. Median age 53 years. $67 \%$ women. All with golimumab $50 \mathrm{mg}$ with an administration interval of 28 to 41 days. Duration of treatment 16.73 months (median). 80\% underwent combination therapy with DMARDs.

Only one patient (RA) presented levels of AcGOL, with undetectable Cmin, DAS28 of 4.05 .

Golimumab levels in patients without AcGOL and DAS28 <3.2: 1 Cmin $<0.25 \mathrm{mcg} / \mathrm{mL}, 5$ Cmin $0.26-0.5 \mathrm{mcg} /$ $\mathrm{mL}, \quad 1$ Cmin $0.5-1.4 \mathrm{mcg} / \mathrm{mL}$ and $12 \mathrm{mcg} / \mathrm{mL}$ Cmin. In patients with DAS28 $>3.2$ were: $2 \mathrm{Cmin}<0.25 \mathrm{mcg} / \mathrm{mL}, 2$ Cmin $0.26-0.5 \mathrm{mcg} / \mathrm{mL}, 2 \mathrm{Cmin} 0.5-1.4 \mathrm{mcg} / \mathrm{mL}$ and $1 \mathrm{Cmin}$ of $2.2 \mathrm{mcg} / \mathrm{mL}$.

Conclusions Very variable GOL Cmin values $(<0.25-2.2 \mathrm{mcg} /$ $\mathrm{mL}$ ) were obtained. $80 \%$ with DAS28 $<3.2$ had a GOL Cm: $0.26-0.5 \mathrm{mcg} / \mathrm{mL}$. Because of the limited information currently available on the optimal range of golimumab levels, further studies are needed to evaluate the relationship between serum drug levels and patient disease activity data.

Disclosure of Interest None declared.

\section{P147 TREATMENT WITH USTEKINUMAB IN PSORIASIC ARTHRITIS IN CONDITIONS OF REAL CLINICAL PRACTICE}

1J Uceda*, ${ }^{2} \mathrm{R}$ Hernandez, ${ }^{3} \mathrm{JL}$ Marenco. 'Rheumatology Unit, University Valme Hospital; ${ }^{2}$ Rheumatology Unit, University Valme Hospital; ${ }^{3}$ Rheumatology Unit, Valme University Hospital, Seville, Spain

\subsection{6/annrheumdis-2018-EWRR2019.130}

Career situation of first and presenting author Assistant.

Introduction Psoriatic arthritis (PsA) belongs to the family of spondyloarthritis, with an incidence of 6 cases per 100000 inhabitants. Ustekinumab is an inhibitor of IL 12/13 approved for patients with PsA who do not respond to disease modifying anti-rheumatic drugs (DMARDs). This drug has been shown to be effective and safe in different clinical trials.

Objectives The primary objective was to assess the effectiveness and safety of Ustekinumab in a cohort of patients with PsA, in follow-up in the Rheumatology Unit of the Valme University Hospital, under conditions of real clinical practice. We have also studied the survival of the drug as well as treatment interruptions and their causes.

Methods We conducted an observational, descriptive, longitudinal and retrospective study of a cohort of 16 patients diagnosed with PsA, according to CASPAR criteria, who receive or have received treatment with Ustekinumab from January 1, 2015 to February 1, 2018.

The effectiveness of the drug was assessed by changing the DAS 28 at 3 and 6 months. We recorded the sociodemographic characteristics of the patients and the characteristics of the disease. Also we recorded previous treatment with anti TNF alpha, and concomitant treatment with DMARDs. We evaluated the suspension of the drug and its reasons. Adverse reactions were recorded too.

Results 16 patients were studied, 56.3\% women. The average age at diagnosis was 41.38 years \pm 8.5 SD. $62.5 \%$ presented polyarticular form, $12.5 \%$ oligoarticular, $6.3 \%$ axial and $18.8 \%$ axial and peripheral arthritis. $87.5 \%$ had rheumatoid factor (RF) and antipeptide-citrullinated antibodies (ACPA) negative. $87.55 \%$ had received prior treatment with at least one antiTNF alpha. $81 \%$ of the sample received Ustekinumab in combination with a DMARs, being methotrexate the most used.

The mean of DAS 28 at the start of treatment with Ustekinumab was $4.51 \pm 1.07 \mathrm{SD}$. The mean of DAS28 was 3.11 $\pm 1.39 \mathrm{SD}$ and $2.93 \pm 1.58 \mathrm{SD}$ after 3 months and 6 months of treatment.

The average survival of ustekinumab in the period analyzed (January 1, 2015 to January 1, 2018) was 29.1 months \pm 3.7 SD.

5 patients of our sample discontinued the treatment: 4 due to ineffectiveness and 1 due to an adverse effect that consisted of a bronchospasm crisis, resolving itself after drug suspension and not requiring hospital admission. There has been no cases of tuberculosis, neoplasia, demyelinating disease or death.

Conclusions Ustekinumab is an effective drug for psoriatic arthritis since three months of treatment. In our study Ustekinumab presented a high survival and a good safety profile in real clinical practice.

Disclosure of Interest None declared. 